\title{
OPTIMIZATION OF THE ELECTRICAL AND MECHANICAL PARAMETERS OF A VIBRATION ENERGY HARVESTER
}

\author{
M. A. Clementino ${ }^{1}$, M. J. Brennan ${ }^{2}$, S. da Silva ${ }^{2}$ \\ ${ }^{1}$ Universidade Estadual do Oeste do Paraná - UNIOESTE, Centro de Engenharias e Ciências \\ Exatas, Campus de Foz do Iguaçu, PR, Brasil (marcelclementino@uol.com.br) \\ ${ }^{2}$ UNESP - Univ Estadual Paulista, Campus de Ilha Solteira, Departamento de Engenharia \\ Mecânica, Ilha Solteira, SP, Brasil
}

\begin{abstract}
The use of piezoelectric energy harvesting devices to power sensor networks and electronic equipment has become popular research topic. For practical use of the energy converted by these transducers conversion of the alternating current (AC) produced to direct current $(D C)$ is generally required. This is normally done by using rectifier circuits. One important issue to overcome is to be able to design a device able to produce the highest power possible. To optimize the coupled mechanical-electrical system a coupled model is required, including the beam structure, piezoelectric element and rectifier circuit, using the same computational software. Thus, for a specific level of mechanical vibration, the maximum power harvested can be obtained by numerical optimization of the beam and piezoelectric geometry and parameters of resistive load, capacitive filter and diodes. All simulations are carried out using Matlab and the optimization is carried out by a sequential quadratic program (SQP) method. The results show which characteristics are important to consider and to modify in order to improve the performance of the power harvesting device.
\end{abstract}

Keywords: energy harvesting, power electronics, unified approach, power optimization.

\section{INTRODUCTION}

In the last twenty years or so, many researchers have been concerned with the development of devices that could increase the lifespan of batteries, as well as reducing the battery replacement rate, by using a process to transform vibration into electrical energy, called energy harvesting. The reduction of energy requirements of some low-powered electronic devices has brought this closer to real applications, especially using piezoelectric materials in energy harvesters, causing a substantial increase in the number of published papers in this area, for example [9,13,15,17,18].

These energy harvesting devices are, in essence, a base structure to which piezoelectric materials (usually PZT ceramics) are attached, for the purposes of generating electrical energy. An electrical circuit then converts the current from alternating current (AC) 
to direct current (DC). Several models have been used to predict the amount of power that these energy harvesters can generate and how much of it can be extracted/stored. Models for the mechanical behavior range from lumped parameter single-degree-of-freedom (SDOF) models [13], to distributed parameters models with Rayleigh-Ritz [16] and finite element approximations [1]. Such papers generally aim to predict the harvester characteristics that lead to the maximum power generation while the energy produced is dissipated by a resistor, which represents the electrical load. However, for practical use of the energy generated by these transducers, the AC produced is generally converted to DC using rectifier circuits. Lallart and Guyomar [10], Guan and Liao [5] and Ottman et al. [11] are a few authors that have modeled advanced harvester circuits (e.g. Synchronized Switch Harvesting on Inductor (SSHI) circuits and rectifier bridges connected to DC/DC converters), and used supercapacitors or rechargeable batteries as storage devices.

Recently, researchers have concentrated their efforts on coupled models i.e., models in which the interaction between both the mechanical structure and electronic circuit is considered. However, many of these models usually simplify either the mechanical domain, considering a SDOF spring-mass-damper models and equivalent circuit techniques $[2,3,20]$, or the electrical domain, using a single resistor to represent the external load. Such models may not correspond to reality. To achieve maximum power generation, several authors have sought the optimal values of structural parameters [4], as well as those related to the electrical circuits [19]. The mechanical parts and the electrical parts of the energy harvesting device are usually modeled in different software packages that may not be compatible. Consequently, the optimization process, which is a very important stage in the design process, can be compromised.

In this paper, the mechanical and electrical parts of the system are modeled using the same software package, which enables the system to be optimized so that the maximum power can be harvested. All the simulations are carried out using Matlab and the optimization process is carried out using a sequential quadratic program (SQP). The paper is organized as follows. Section 2 presents the piezoelectric harvester device and harvesting circuit models. Section 3 describes in detail the optimization problem. The results are presented in Section 4, which is followed by some conclusions in Section 5.

\section{MODEL OF THE PIEZOELECTRIC HARVESTING DEVICE COUPLED WITH THE ENERGY EXTRACTION CIRCUIT}

The electro-mechanical generating device consists of a cantilever beam to which two piezoelectric elements are attached. It is shown in Fig. (1), where $L_{b}$ and $L_{p}$ are the length of the beam and Piezoelectric elements, respectively; $t_{b}$ and $t_{p}$ are their respective thicknesses; $w$ is the width of both elements; $L_{x}$ is the distance between the piezoelectric elements and the clamped end; the subscripts 1 and 2 stand for the upper and lower piezoelectric elements respectively.

The model is based on that proposed by Sodano et al. [16], which has been used frequently due to its complete formulation that accounts for multiple modes. This paper highlights only the main points of their work, since a detailed description of the model can be 
found in [16]. The model is developed using Hamilton's variational principle with the piezoelectric linear properties, while some additional assumptions are made. First, Euler-Bernoulli beam theory is considered, which means the model is limited to a system with small displacements and strain, and linear behavior. Second, it is assumed that the piezoelectric elements are perfectly bonded to the surface of the beam, and the electric voltage $v(t)$ produced is constant over the thickness of the piezoelectric elements.



Figure 1. Schematic of the beam of the energy harvesting device with two piezoelectric patches attached.

The piezoelectric behavior is described by [8]:

$$
\left\{\begin{array}{l}
T_{1} \\
D_{3}
\end{array}\right\}=\left[\begin{array}{cc}
c_{11}^{E} & -e_{31} \\
e_{31} & \epsilon_{33}^{S}
\end{array}\right]\left\{\begin{array}{l}
S_{1} \\
E_{3}
\end{array}\right\} .
$$

Where $T$ is the stress tensor $\left[\mathrm{N} / \mathrm{m}^{2}\right], S$ is the strain tensor $[\mathrm{m} / \mathrm{m}], E$ is the electric field $[\mathrm{N} / \mathrm{C}], D$ is the electric displacement $\left[\mathrm{C}^{2} \mathrm{~m}^{2}\right], c^{E}$ is the modulus of elasticity at a constant electric field $\left[\mathrm{N} / \mathrm{m}^{2}\right], \epsilon^{S}$ is the dielectric constants matrix at constant strain and $e$ is the piezoelectric coupling coefficient, that relates the stress to the applied electric field. Generally, the piezoelectric constant $d=\frac{e}{c^{E}}$ is used in the modeling, since this property is usually provided by manufacturers.

Once the piezoelectric properties are known and their effects are considered using Hamilton's principle, the next step involves solving the resulting equation. However, as the beam is a continuous system, finding a solution to its governing equation requires the use of an approximate method. A common approach to this is to use the Rayleigh-Ritz method, which states that the displacement of the system can be represented by the sum of a finite number of modes, $n$, each with mode-shape $\boldsymbol{\Phi}_{n}(x)$. Hence, the equation of motion for the beam with the piezoelectric elements attached is given by $[6,14,16]$ :

$$
\begin{gathered}
\left(\mathbf{M}_{\mathbf{s}}+\mathbf{M}_{\mathbf{p}}\right) \ddot{\mathbf{r}}(t)+\mathbf{C r}(t)+\left(\mathbf{K}_{\mathbf{s}}+\mathbf{K}_{\mathbf{p}}\right) \mathbf{r}(t)+\boldsymbol{\Theta} v(t)=\mathbf{F}(t) \\
-\boldsymbol{\Theta}^{T} \dot{\mathbf{r}}(t)+\mathbf{C}_{\mathbf{p}} \dot{v}(t)=-\dot{q}(t) .
\end{gathered}
$$

where $\mathbf{M}$ and $\mathbf{K}$ are the mass and stiffness matrices, respectively; $\mathbf{C}$ is the damping matrix, assumed to be proportional to mass and stiffness by the constants $\alpha$ and $\beta$, respectively; $\boldsymbol{\Theta}=\boldsymbol{\Theta}^{T}$ is the electromechanical coupling matrix; $\mathbf{C}_{\mathbf{p}}$ is the piezoelectric capacitance; the 
subscripts $s$ and $p$ represent the structural and piezoelectric elements, respectively; $\mathbf{r}(t)$ is the displacement; $q(t)$ is the electric charge. The dot over the variables $v(t), q(t)$ and $\mathbf{r}(t)$ stand for the time derivatives, where $\dot{q}(t)$ is the electric current $i(t)$. As shown in Fig. (1), the beam is subjected to an input displacement at the base of the beam. This can be approximately represented by a force at the tip of the beam, given by

$$
F(t)=\int_{V} \rho A \omega^{2} \sin (\omega t) d V=F_{o} \sin (\omega t)
$$

where $F_{o}=\int_{V} \rho_{b} A \omega^{2} d V$ is the force amplitude, $\omega$ is the excitation frequency, $A$ is the amplitude of the displacement at the base of the beam, $V$ is the volume and $\rho_{b}$ is the density of the beam. The force $F(t)$ is calculated assuming only the mass of the beam, i.e. neglecting the mass of the piezoelectric elements.

Equations (2) and (3) can only represent a real Power Harvesting system, if the connection between the piezoelectric beam and the rectifier circuit is taken into account. Figure (2) illustrates this connection, where a full wave diode bridge rectifier circuit is considered, with a capacitive filter $C_{L}$ and a resistive load $R_{L}$ that represents the external load.

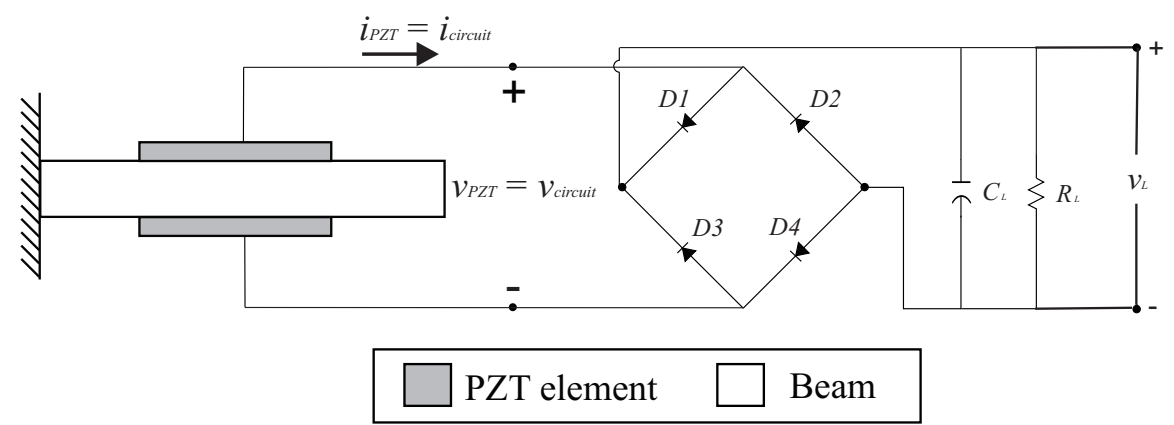

Figure 2. Schematic diagram of the coupling between the piezoelectric harvester and the full wave diode bridge rectifiers.

According to Rupp et al. [14], these two systems may be coupled assuming a continuity condition between the current/voltage of the mechanical and electrical domains, which means that $v_{\text {circuit }}=v_{\mathrm{PZT}}$ and $i_{\text {circuit }}=i_{\mathrm{PZT}}$. In addition, in eq. (3) $\dot{q}(t)$ represents the current passing through the rectifier circuit and, consequently, feeds back the piezoelectric element. The diodes used in the circuit can be described by an equivalent resistance $R_{D}$ and a forward voltage drop $v_{f d}$, since a piecewise linear model is used. Thus, due to the capacitive element, if $|v(t)|<2 v_{f d}+v_{L}(t)$, no current flows and $v_{L}(t)$ is the voltage across the load. In this case, $i(t)=0$ and $v_{L}(t)$ decreases while the capacitor supplies the resistive load according the equation:

$$
C_{L} \dot{v}_{L}(t)+\frac{v_{L}(t)}{R_{L}}=0
$$

When $v(t) \geq 2 v_{f d}+v_{L}(t)$, the current $i(t)$ that flows through the diodes $D_{1}$ and $D_{4}$ becomes: 


$$
i(t)=\frac{v(t)-v_{L}(t)-2 v_{f d}}{R_{D}}
$$

Hence, the voltage across the load is now described by

$$
C_{L} \dot{v}_{L}(t)+\left(\frac{1}{R_{L}}+\frac{1}{2 R_{D}}\right) v_{L}(t)=\frac{v(t)-2 v_{f d}}{2 R_{D}} .
$$

Likewise in this case, when $-v(t) \geq 2 v_{f d}+v_{L}(t)$, for the negative half cycle, the current flowing through the diodes $D_{2}$ and $D_{3}$ is:

$$
i(t)=\frac{v(t)+v_{L}(t)+2 v_{f d}}{R_{D}}
$$

Hence, the voltage $v_{L}(t)$ across the load is described by

$$
C_{L} \dot{v}_{L}(t)+\left(\frac{1}{R_{L}}+\frac{1}{2 R_{D}}\right) v_{L}(t)=-\frac{v(t)+2 v_{f d}}{2 R_{D}} .
$$

The eqs. (5) to (9) represent the full wave diode bridge rectifier circuit in terms of the variables $v(t), i(t)$ and $v_{L}(t)$, where the latter can be used to obtain the power harvested. In this paper, the root mean square of the power $P_{r m s}$ is used and is calculated by

$$
P_{r m s}=\frac{v_{r m s}^{2}}{R_{L}}
$$

where $v_{r m s}=\sqrt{v_{L}(t)^{2} / t_{f}}$ and $t_{f}$ is the time over which the calculation is made.

A convenient way to describe the complete electro-mechanical model of the energy harvesting device is to use a state-space model, which is given by

$$
\begin{gathered}
\dot{\mathbf{x}}=\mathbf{A} \mathbf{x}+\mathbf{B}_{1} u_{1}+\mathbf{B}_{2} u_{2} \\
\mathbf{y}=\mathbf{C}_{o} \mathbf{x}+\mathbf{D}_{1} u_{1}+\mathbf{D}_{2} u_{2}
\end{gathered}
$$

where $\mathbf{x}=\left\{\begin{array}{lll}\mathbf{r}(t) & \dot{\mathbf{r}}(t) & v(t)\end{array}\right\}^{T}$ is the state vector and $u_{1}$ and $u_{2}$ are the inputs of the system. The dynamic matrix $\mathbf{A}$, the input matrix $\mathbf{B}$, the output matrix $\mathbf{C}_{o}$ and the direct transmission matrix $\mathbf{D}$ are given by:

$$
\begin{gathered}
\mathbf{A}=\left[\begin{array}{ccc}
\mathbf{0}_{n \times n} & \mathbf{I}_{n \times n} & \mathbf{0}_{n \times 1} \\
-\mathbf{M}^{-1} \mathbf{K} & -\mathbf{M}^{-1} \mathbf{C} & -\mathbf{M}^{-1} \boldsymbol{\Theta} \\
\mathbf{0}_{1 \times n} & C_{p}^{-1} \boldsymbol{\Theta}^{T} & 0
\end{array}\right], \quad \mathbf{B}_{1}=\left[\begin{array}{c}
\mathbf{0}_{n \times 1} \\
\mathbf{M}^{-1} \mathbf{F} \\
0
\end{array}\right], \quad \mathbf{B}_{2}=\left[\begin{array}{c}
\mathbf{0}_{n \times 1} \\
\mathbf{0}_{n \times 1} \\
C_{p}^{-1}
\end{array}\right] \\
\mathbf{C}_{o}=\left[\begin{array}{ccc}
\mathbf{0}_{1 \times n} & \mathbf{0}_{1 \times n} & 1 \\
\boldsymbol{\Phi} & \mathbf{0}_{1 \times n} & 0
\end{array}\right], \quad \mathbf{D}_{1}=0, \quad \mathbf{D}_{2}=0
\end{gathered}
$$


where $\boldsymbol{\Phi}=\left\{\Phi_{1}(x) \quad \Phi_{2}(x) \cdots \Phi_{n}(x)\right\}$ is the vector of mode-shapes used to approximate the transversal displacement $\mathbf{u}(x, t), \mathbf{0}$ is the zero matrix and $\mathbf{I}$ is the identity matrix. The output matrix $\mathbf{C}_{o}$ gives the output of the electromechanical system $\mathbf{y}=\left\{\begin{array}{lll}v(t) & \dot{\mathbf{u}}(x, t) \quad \mathbf{u}(x, t)\end{array}\right\}^{T}$. The input vector is defined by $u_{1}=\sin (\omega t)$ and $u_{2}=-\dot{q}(t)$, where $u_{1}$ must be multiplied by $F_{0}$ to produce the applied external force. The feedback current $u_{2}$ is negative because of the energy extraction effect, which is why the variable on the right-hand side of eq. (3) has a negative sign. With the system represented as a space-state model, it is possible to perform an optimization of the complete system. All simulations are carried out within Matlab, where the statespace and the rectifier circuit models are implemented using the Simulink toolbox.

\section{OPTIMIZATION THE ENERGY HARVESTER}

The goal of this paper is to maximize the power $P_{r m s}$ generated by the piezoelectric harvester using the Sequential Quadratic Programming (SQP) optimization method. To achieve this, the objective function which was the inverse of $P_{r m s}$ is minimized. The optimization parameters considered are the length of the beam $\left(L_{b}\right)$ and piezoelectric elements $\left(L_{p}\right)$, the resistive load $\left(R_{L}\right)$, the capacitor $\left(C_{L}\right)$ and the diodes parameters $R_{D}$ and $v_{f d}$.

As the capacitor included in the rectifier circuit is required to store the energy harvested, this energy must be maximized, and is another requirement that must be taken into account. Thus, the energy stored in the capacitor given by

$$
E_{C}=\frac{1}{2} C_{L} v_{L}^{2}
$$

must also be considered as an objective function. Hence, the constrained minimization problem can be written as:

$$
\text { Minimize } f(x)=\frac{1}{P_{r m s}} \text { and } h\left(v_{L}, C_{L}\right)=\frac{1}{E_{C}}
$$

where the vector of variables to be optimized is

$$
\mathbf{x}=\left\{\begin{array}{llllll}
L_{b} & L_{p} & R_{L} & C_{L} & R_{D} & v_{f d}
\end{array}\right\}^{T}
$$

subject to the constraints:

$$
\begin{array}{ll}
g_{1}(x): L_{b}^{\min } \geq L_{b} \geq L_{b}^{\max } & g_{2}(x): L_{p}^{\min } \geq L_{p} \geq L_{p}^{\max } \\
g_{3}(x): R_{L}^{\min } \geq R_{L} \geq R_{L}^{\max } & g_{4}(x): C_{L}^{\min } \geq C_{L} \geq C_{L}^{\max } \\
g_{5}(x): R_{D}^{\min } \geq R_{D} \geq R_{D}^{\max } & g_{6}(x): v_{f d}^{\min } \geq v_{f d} \geq v_{f d}^{\max }
\end{array}
$$

The optimization parameters are thus each restricted to a range of feasible values. 


\section{RESULTS}

The optimization procedure was applied to the system discussed under harmonic base excitation. Table (1) gives the dimensions and properties of the model electromechanical system. The simulations were carried out considering four mode shapes $(N=4)$ of the beam, with $f=\omega /(2 \pi)=70 \mathrm{~Hz}$ as the excitation frequency and $A=1.53 \times 10^{-4} \mathrm{~m}$ as the displacement amplitude. Such parameters ( $\omega$ and $A$ ) were chosen to represent real operation conditions, since the piezoelectric harvester are usually placed in locations containing these characteristics. The optimization results are shown in Fig. (3) and Tab. (2), where the initial conditions and the constraints used in the procedure are also presented.

Table 1. Parameters of the piezoelectric harvester model.

\begin{tabular}{ccc}
\hline Domain & Parameters & Values \\
\hline \multirow{3}{*}{ Structural element } & Thickness $\left(t_{b}\right)$ & $0.91 \mathrm{~mm}$ \\
& Width $(w)$ & $21 \mathrm{~mm}$ \\
& Density $\left(\rho_{b}\right)$ & $2700 \mathrm{~kg} / \mathrm{m}^{3}$ \\
& Modulus of elasticity $\left(E_{b}\right)$ & $70 \mathrm{Gpa}$ \\
& Material & Aluminum \\
\hline \multirow{3}{*}{ Piezoelectric element } & Thickness $\left(t_{p}\right)$ & $0.191 \mathrm{~mm}$ \\
& Density $\left(\rho_{p}\right)$ & $7750 \mathrm{~kg} / \mathrm{m}^{3}$ \\
& Modulus of elasticity $\left(E_{p}\right)$ & $60.60 \mathrm{Gpa}$ \\
& Model & PZT-5H \\
& Dielectric constant $\left(K_{33}\right)$ & 3400 \\
& Piezoelectric constant $\left(d_{31}\right)$ & $274 \times 10^{-12} \mathrm{~m} / \mathrm{V}$ \\
\hline
\end{tabular}

Scaled parameters are shown in Fig. (3) because their magnitudes are very different. The parameters were scaled according to: $C_{L}=1 \times 10^{-5} \mathrm{~F}, R_{L}=1 \times 10^{3} \Omega, L_{b}=L_{p}=2 \times 10^{-3} \mathrm{~m}$ , $R_{D}=5 \Omega, v_{f d}=1 \times 10^{-3} \mathrm{~V}$. The results in Tab. (2) show the optimized results as well as the ranges considered in the search for the optimum parameters

Table 2. Results of the optimization procedure.

\begin{tabular}{ccccc}
\hline \multirow{2}{*}{ Parameters } & \multirow{2}{*}{ Lower Bound } & \multirow{2}{*}{ Upper Bound } & \multicolumn{2}{c}{ Configuration } \\
\cline { 3 - 5 } & & $1 \times 10^{-3}$ & $0.17 \times 10^{-3}$ & $0.201 \times 10^{-3}$ \\
$C_{L}[\mathrm{~F}]$ & $1 \times 10^{-6}$ & $1 \times 10^{5}$ & $15 \times 10^{3}$ & $62.52 \times 10^{3}$ \\
$R_{L}[\Omega]$ & $1 \times 10^{-3}$ & $150 \times 10^{-3}$ & $53 \times 10^{-3}$ & $66.4 \times 10^{-3}$ \\
$L_{b}[\mathrm{~m}]$ & $50 \times 10^{-3}$ & $150 \times 10^{-3}$ & $127.5 \times 10^{-3}$ & $130.2 \times 10^{-3}$ \\
$L_{p}[\mathrm{~m}]$ & $50 \times 10^{-3}$ & 500 & 280 & 280.66 \\
$R_{D}[\Omega]$ & 50 & $100 \times 10^{-3}$ & $50 \times 10^{-3}$ & $48.4 \times 10^{-3}$ \\
$v_{f d}[\mathrm{~V}]$ & $30 \times 10^{-3}$ & - & $88.04 \times 10^{-3}$ & $92.95 \times 10^{-3}$ \\
\hline Power RMS $[\mathrm{W}]$ & - & & & \\
\hline
\end{tabular}






Figure 3. Evolution of the scaled optimization parameters in the optimization procedure iterations.

It can be seen from Fig. (3) that the design parameters reach their optimum values after about 20 iterations. Further, it can be seen from Tab. (3) that the rms value of the power harvested was increased from the initial design by about 5.3\%. For some parameters, the optimal values differ little from the chosen initial condition, suggesting that the optimization algorithm may not have sought the optimal solution through the whole domain.

This could be because the Simulink Design Optimization tool does not assure a global optimal solution, or it could be that the initial values were quite close to the optimal values. Comparing the optimal configuration to a similar one, with the same values but setting $R_{D}$ and $v_{f d}$ to $65 \Omega$ and $38 \times 10^{-3} \mathrm{~V}$, respectively, it could be seen that the solution is not really the global maximum.

Figure (5) presents the way in which the values of the design parameters and the excitation frequency affect the power harvested. Examining this figure it can be seen that all optimal values remains inside a region where the power is maximum, i.e. around $70 \mathrm{~Hz}$. Since the length of the beam greatly influences the natural frequency of the system, the maximum power varies dramatically with the length, as can be seen in Fig. (5(b)). The optimal values of the electrical parameters also occur around the excitation frequency. Figures (5(c)) and (5(d)) show that $C_{L}$ and $R_{L}$, have a large effect on the amount of power that can be harvested and are thus important to consider in the optimization process.

\section{CONCLUSIONS}

The use of piezoelectric devices to harvest vibration energy has been considered in this paper. A unified model of a beam with piezoelectric elements attached connected to a full wave diode bridge rectifier circuit has been developed. This coupled electro-mechanical model has enabled the power harvested to be maximized by optimizing both mechanical and elec- 
trical parameters. Despite the fact that the optimization tool does not assure a global maximum solution, the results showed that when the diode is represented as a linear equivalent model, its characteristics do not cause large changes to the power harvested. However, the mechanical parameters are important to ensure that the natural frequency of the beam is coincident with the excitation frequency. It was also shown that the values of capacitor and the load resistor in the electrical circuit are also vitally important in an optimally designed power harvester.

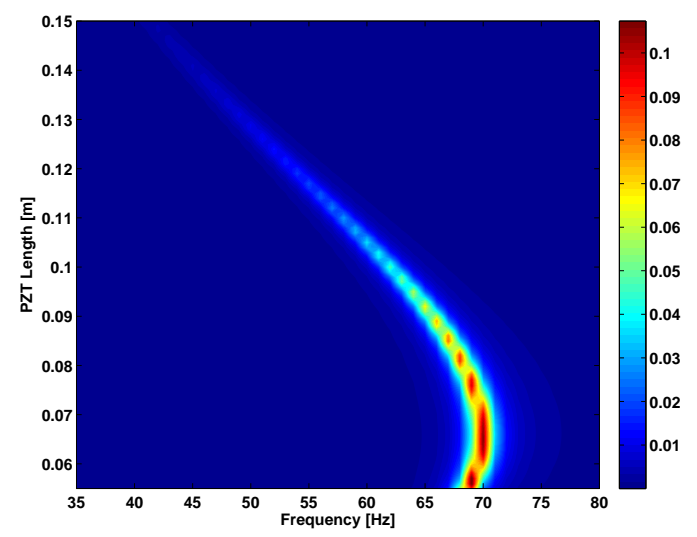

(a) Power as a function of piezoelectric element length and excitation frequency.

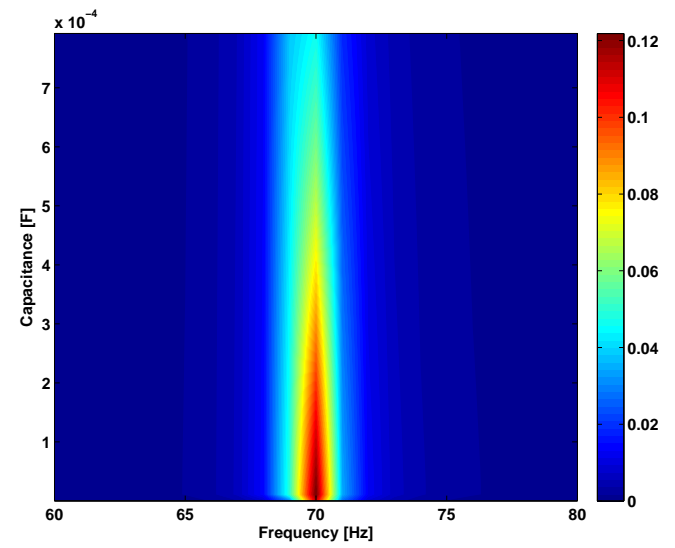

(c). Power as a function of capacitance and excitation frequency.

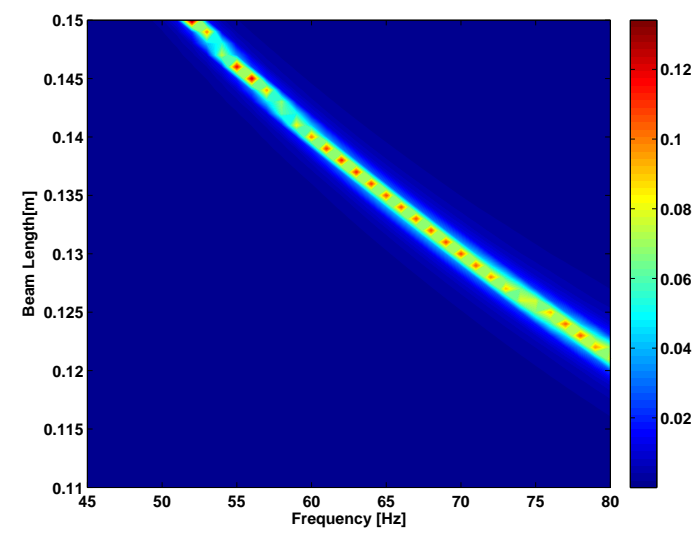

(b). Power as a function of beam length and excitation frequency.

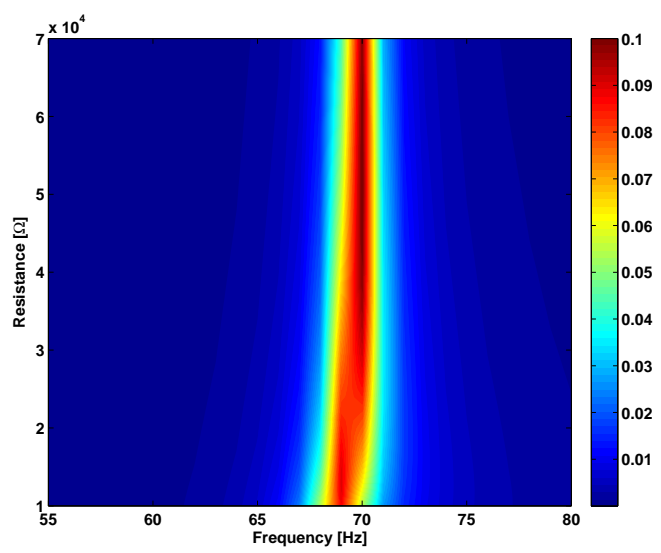

(d). Power as a function of resistive load and excitation frequency.

Figure 5. Influence of $L_{p}, L_{b}, C_{L}$ and $R_{L}$ on power harvested and the excitation frequency.

\section{Acknowledgements}

The first author would like to thank the CAPES for his scholarship. The authors are also thankful the financial support provided by National Council for Scientific and Technological 
Development (CNPq), Fundação Araucária de Desenvolvimento Científico e Tecnológico do Paraná (PR) and Fundação de Amparo à Pesquisa do Estado de São Paulo (FAPESP/SP).

\section{REFERENCES}

[1] de Marqui Jr. C., Erturk A. and Inman D. J., "An electromechanical finite element model for piezoelectric energy harvester plates". Journal of Sound and Vibration, 327, 9-25, 2009.

[2] Elvin N. G. and Elvin A. A., "A general equivalent circuit model for piezoelectric generators". Journal of Intelligent Material Systems and Structures, 20, 3-9, 2008.

[3] Elvin N. G. and Elvin A. A., "A coupled finite element-circuit simulation model for analyzing piezoelectric energy generator". Journal of Intelligent Materials Systems and Structures, 20, 587-595, 2009.

[4] Franco V. R., Mineto A. T. and Varoto P. S., "Optimization strategies for optimum power generation in piezoelectric energy harvesting from ambient vibrations". In 21st International Congress of Mechanical Engineering, (Natal, RN, Brazil), UFRN/ABCM, 2011.

[5] Guan M. J. and Liao W. H., "On the efficiencies of piezoelectric energy harvesting circuits towards storage device voltages". Smart Materials and Structures, 16, 498-505, 2007.

[6] Hagood N. W., Chung W. H. and Flotow A. V., "Modeling of piezoelectric actuator dynamics for active structural control". Journal of Intelligent Materials Systems and Structures, 1, 327-354, 1990.

[7] Hu Y., Hu T. and Jiang Q., "On the interaction between the harvesting structure and the storage circuit of a piezoelectric energy harvester". International Journal of Applied Electromagnetics and Mechanics, 27, 297-309, 2008.

[8] IEEE, "IEEE standard on piezoelectricity". 1987.

[9] Kymissis J., Kendall C., Paradiso J. and Gershenfeld N., "Parasitic power harvesting in shoes". Second International Symposium on Wearable Computers, 132-139, 1998.

[10] Lallart M. and Guyomar D., "An optimized self-powered switching circuit for nonlinear energy harvesting with low voltage output". Smart Materials and Structures, 17, 035030, 2008.

[11] Ottman G. K., Hofmann H. F., Bhatt A. C. and Lesieutre G. A., "Adaptive piezoelectric energy harvesting circuit for wireless remote power supply". IEEE Transactions on Power Electronics, 17, 669-676, 2002.

[12] Renno J. M., Daqaq M. F. and Inman D. J., "On the optimal energy harvesting from a vibration source". Journal of Sound and Vibration, 320, 386-405, 2009. 
[13] Roundy S., Wright P. K. and Rabaey J., "A study of low level vibrations as a power source for wireless sensor nodes". Computer Communications, 26, 1131-1144, 2003.

[14] Rupp C. J., Dunn M. L. and Maute K., "Analysis of piezoelectric energy harvesting systems with non-linear circuits using the harmonic balance method". Journal of Intelligent Material Systems and Structures, 21, 1383-1396, 2010.

[15] Shenck N. S. and Paradiso J. A., "Energy scavenging with shoe-mounted piezoelectrics". IEEE Micro, 21, 30-42, 2001.

[16] Sodano H. A., Inman D. J. and Park G., "Estimation of electric charge output for piezoelectric energy harvesting". Strain, 40, 49-58, 2004.

[17] Sodano H. A., Inman D. J. and Park G., "Comparison of piezoelectric energy harvesting devices for recharging batteries". Journal of Intelligent Material Systems and Structures, 16, 799-807, 2005.

[18] Starner T., "Human-powered wearable computing". IBM Systems Journal, 35, 618-629, 1996.

[19] Wickenheiser A. M. and Garcia E., "Power optimization of vibration energy harvesters utilizing passive and active circuits". Journal of Intelligent Material Systems and Structures, 21, 1343-1361, 2010.

[20] Yang Y. and Tang L., "Equivalent circuit modeling of piezoelectric energy harvesters". Journal of Intelligent Materials Systems and Structures, 20, 2223-2235, 2009. 